\title{
An indirect method of measuring perceived distance from familiar size
}

\author{
WALTER C. GOGEL \\ University of California, Santa Barbara, California 93106
}

\begin{abstract}
Two methods of measuring perceived distance as a function of familiar size were compared in five experiments. The method which uses the perception of motion concomitant with a motion of the head, unlike the method of verbal report, is considered to provide a measure of perceived distance that is unaffected by factors of cognitive distance. The results of the experiments indicate that although the perceived egocentric distance of an object can vary somewhat as a function of the cue of familiar size, the larger variation often found with verbal reports of distance is based upon cognitive, not perceptual, information. The cognitive information is interpreted as resulting from the perception of the object as off-sized and the observer's assumption that the perceived size of an object will vary inversely with its physical distance.
\end{abstract}

The possibility that familiar size can provide a cue to the distance of the familiar object from the observer (perceived egocentric distance) is of particular significance for theories of space perception. There are several reasons for the theoretical importance of this cue. First, familiar size is the only cue to egocentric distance which, by definition, supports the empiricistic position that past experience is necessary for the perception of distance. The second reason concerns the magnitude of perceived distance produced by egocentric cues. The egocentric cues of convergence, accommodation, and absolute motion parallax, if effective, are effective only for distances within several meters from the observer. The familiar size cue to egocentric distance, however, has the potential of specifying the perceived distances of objects far from the observer. The reason for this follows from a consideration of the familiar size cue to distance as a special case of the size-distance invariance hypothesis (SDIH). The SDIH often is expressed as

$$
S^{\prime}=K_{1} \theta D^{\prime},
$$

where $S^{\prime}$ and $D^{\prime}$ are the perceived size and perceived egocentric distance, respectively, of an object of retinal size $\theta$ with $K_{1}$ an observer constant. According to Equation 1, if the known size of a familiar object is able to determine a perceived size, it also will be able to determine a perceived distance with the magnitude of the perceived distance directly related to $\mathrm{S}^{\prime} / \theta$, the ratio of perceived to retinal size. Thus, the familiar size of an object, regardless of whether

This investigation was supported by PHS Research Grant MH-15651 from the National Institute of Mental Health. The author wishes to thank Robert E. Newton for his help with the apparatus and data collection. the object is near or far, can provide a cue to distance whenever the physical size of the object at that distance is sufficient to produce a retinal size that is above the threshold of detection. It follows that the familiar size cue to distance is a potentially important source of information regarding egocentric distance throughout the visual field.

The question of whether familiar size is an effective cue to perceived egocentric distance has produced a considerable amount of research (see Epstein, 1967). It seems clear from this research that, although the precision of this cue is not impressive, the reported distance of a familiar object will vary in a manner consistent with Equation 1. But, in order to be entirely consistent with Equation 1, the reported distances must not only vary directly with familiar and inversely with retinal size but it must be demonstrated that the response being measured is a perceptual as contrasted with a cognitive (inferred) response of distance. It is this latter problem that will be examined in the present study.

A test of whether familiar size is an effective cue to perceived distance requires that other distance cues which might determine the perceived distance are reduced or eliminated. According to the SDIH, if familiar size under these conditions is to be a cue to perceived distance, it must be a cue to perceived size. But there is considerable evidence that in situations in which other distance cues are reduced, familiar size does not uniquely determine perceived size (Epstein, 1961; Gogel, 1969; Gogel \& Mertens, 1967; Gogel \& Newton, 1969). In these experiments, rather than perceiving the single, familiar object presented monocularly (usually in an otherwise dark environment) as having a size that is normal for that object, the object often is reported as being smaller or larger than normal. It is suggested that this perception of familiar objects as off-sized can result 
from the tendency to perceive the objects at about 2 or $3 \mathrm{~m}$ from the observer (the specific distance tendency) whenever this distance is different from the perceived distance expected from familiar size (Gogel, 1974). It has been shown that if the distance expected from the familiar size cue is greater or less than the distance of the specific distance tendency, the familiar object will be perceived as smaller or larger than normal, respectively (Gogel, 1969; Gogel \& Newton, 1969). This research also indicates that the perception of the familiar object as off-sized provides the observer with a cognitive option in his judgment of distance. This cognitive option is based upon the observer's notion that the perceived size of an object of constant physical size will decrease with increasing distance from the observer (Carlson, 1960, 1962; Epstein, 1963). Conversely, if the observer perceives the familiar object as nonnormal in size, this can be used to infer that the object is at a different distance than the distance at which it appears. For example, a familiar object that is perceived to be twice or one-third as large as normal would be inferred to be at one-half or three times the distance from the observer, respectively, as the distance at which it appears. A generalization of this relation between off-sized perceptions and cognitive distance is that

$$
\mathrm{D}_{\mathrm{c}}=\mathrm{K}_{\mathbf{2}} \mathrm{D}^{\prime}\left(\mathrm{S}_{\mathrm{c}} / \mathrm{S}^{\prime}\right) \text {, }
$$

where $D_{c}$ is the inferred or cognitive distance of the object, $\mathrm{S}_{\mathrm{c}}$ is its known, cognitive, or familiar size, $K_{2}$ is an observer constant, and $S_{c} / S^{\prime}$ is the off-sized perception (Gogel, 1974). The object will be perceived as a large off-sized object if $S_{c}<S^{\prime}$, as a small off-sized object if $S_{c}>S^{\prime}$, and as a normal sized object if $S_{c}=S^{\prime}$. Substituting the expression for $S^{\prime}$ from Equation 1 in Equation 2 with $K_{1} / K_{2}=K_{3}$, the relation between $S_{c}$ and $D_{c}$ becomes

$$
\mathrm{S}_{\mathrm{c}}=\mathbf{K}_{\mathbf{3}} \theta \mathrm{D}_{\mathrm{c}} \text {. }
$$

It will be noted that Equations 1 and 3 are similar in form but not in meaning. Equation 1 refers to perceived extents ( $S^{\prime}$ and $D^{\prime}$ ), while Equation 3 refers to cognitive extents $\left(S_{c}\right.$ and $\left.D_{c}\right)$. Because it is difficult for the experimenter to know whether the responses are being determined by cognitive or by perceptual factors, it is often difficult to know whether the data apply to Equation 2 or Equation 3.

Since the forms of Equations 1 and 3 are identical, it is reasonable to question whether it is necessary to distinguish between perceptual and cognitive sources of spatial information. One portion of the answer is that the psychological processes underlying Equations 1 and 3 are likely to be different. For example, the processes underlying Equation 1 may be innately determined, whereas those underlying Equation 3 obviously involve learning. A reason more relevant to the validity of Equations 1 and 3 is that mixtures of perceptual and cognitive sources of information can produce data that mistakenly would seem to disaffirm either of these equations. For example, according to Equation $2, D_{c}$ is proportional to $\mathrm{D}^{\prime}$ only if $\mathrm{S}_{\mathrm{c}}$ is proportional to $\mathrm{S}^{\prime}$. In situations in which this is not the case, neither Equation 1 nor Equation 3 will fit the response data if the responses to size and distance are determined, respectively, by $D_{c}$ and $S^{\prime}$ or by $D^{\prime}$ and $S_{c}$. Consider more generally the situation in which differing cognitive and perceptual information $\left(S_{c} \neq S^{\prime}\right.$ and $\left.D_{c} \neq D^{\prime}\right)$ is available for the observer's verbal report of the apparent size and apparent distance of an object. In verbally reporting the apparent size ( $\left.S^{\prime}{ }_{v}\right)$ of the object, either $S_{c}$ (as determined, for example, by familiar size) or $S^{\prime}$ (as determined by $D^{\prime}$ and $\theta$ in Equation 1), or some weighted average of $S_{c}$ and $S^{\prime}$, might be used by the observer, i.e.,

$$
S^{\prime}{ }_{v}=a S^{\prime}+(1-a) S_{c}
$$

where $\mathbf{a}$ and $1-a$ are the relative weights given to the perceived and cognitive sources of information regarding size. In verbally reporting the apparent distance $\left(D^{\prime} v\right.$ ) of the object, either $D_{c}$ (as specified by Equation 2) or $\mathrm{D}^{\prime}$ (as specified by Equation 1), or some weighted average of $D_{c}$ and $D^{\prime}$, might be used, i.e.,

$$
D^{\prime}{ }_{v}=b D^{\prime}+(1-b) D_{c},
$$

where $b$ and $1-b$ are the relative weights given to the perceived and cognitive sources of information regarding distance. The experimenter, because he asks the observer to report the apparent size and apparent distance of the object, is apt to assume that $S^{\prime}{ }_{v}=S^{\prime}$ and that $D^{\prime}{ }_{v}=D^{\prime}$. But, if $a$ and $b$ are not unity, not only will the application of the results as a test of Equation 1 be inappropriate, but the computed value of $K_{1}$ will vary as a function of the values of $a$ and $b$. This could result in $K_{1}$ varying between experiments. Also, if $a$ and $b$ are different functions of the stimulus conditions, a plot of the $D^{\prime}{ }_{v}$ and $S^{\prime}{ }_{v} / \theta$ data would be nonlinear and the experimenter would conclude erroneously that Equation 1 is incorrect in form.

The present study differentiates between $D^{\prime}$ and $D_{c}$ in tests of the familiar size cue of distance by comparing the distance responses obtained by two methods of measuring perceived egocentric distance as a function of familiar size. One method, a headmotion method, is an instance of an indirect method, and the other, that of verbal reports, is an instance of a direct measure of perceived distance. Direct 
methods or direct procedures for measuring perceived characteristics require that the observer respond quantitatively in terms of the perceptual dimension being measured. In direct measures, the observer's response may be and often is in a different modality than the perception being measured. The distinguishing characteristic, of all direct measures of perception, however, is that the perception being measured by the experimenter and the perception to which the observer is responding are isomorphic. The verbal report of the distance of the object in feet or inches is a direct method of

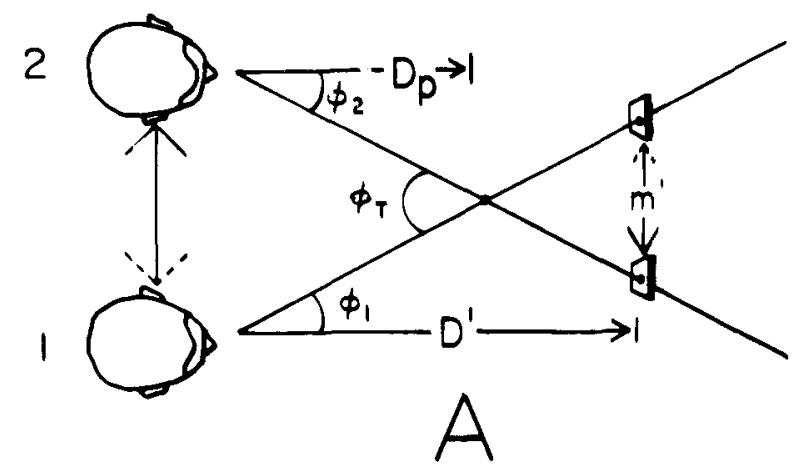

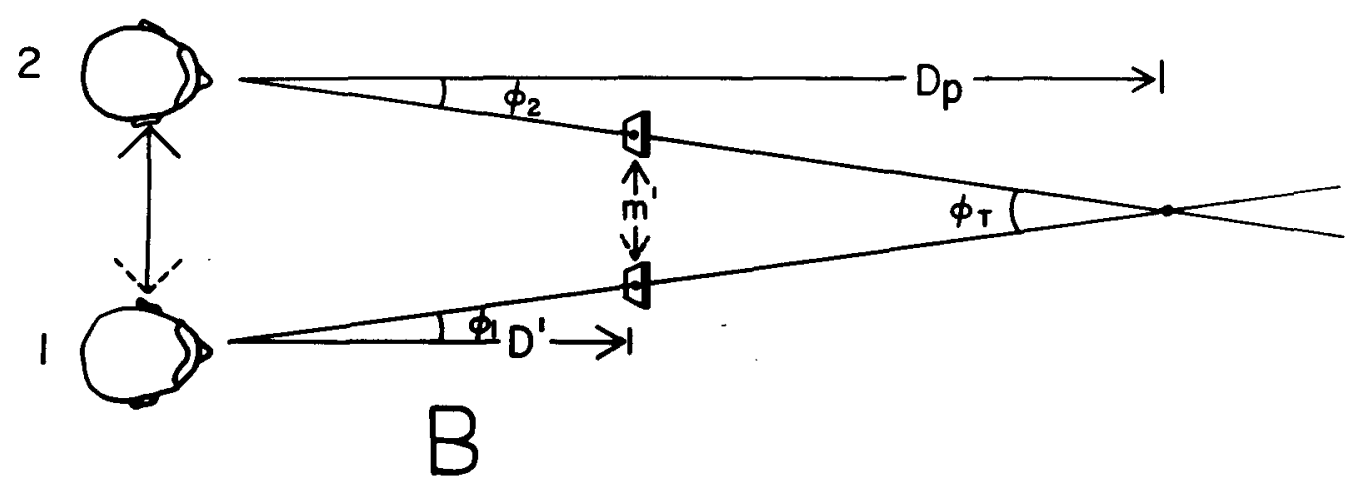

Figure 1. Perceived motion $\left(\mathrm{m}^{\prime}\right)$ concomitant with head motion of a rectangularly shaped object at a perceived distance $\left(D^{\prime}\right)$ as a function of the pivot distance $\left(D_{p}\right)$.

measuring the perceived distance of the object. Other examples of direct methods are the throwing of darts to the apparent distance of the object (Gogel, Hartman, \& Harker, 1957) or the amount of arm extension considered by the observer to be necessary in order to reach to the distance of the object (Foley \& Held, 1972). Indirect methods measure the observer's perception on one dimension by his direct response to another perceptual dimension which from the observer's point of view is unrelated to the perception being measured. Indeed, with an indirect method, it is unnecessary to inform the observer of the dimension of interest to the experimenter. It is necessary, however, that the experimenter know the relation between the two perceptual dimensions. The indirect method used in the present study is illustrated in Figure 1. The diagrams of Figure 1 represent top-view drawings of a situation in which the observer moves his head laterally between Positions 1 and 2 while fixating a single object (rectangle) physically located along a rigid rod extending to the position of the observer's head. The angle through which the head or eyes must turn in order to fixate the object is indicated as $\phi_{T}$, where $\phi_{\mathrm{T}}=\phi_{1}+\phi_{2}$. The apparent egocentric distance of the rectangle (not its physical position) along this rod is the variable measured by this method, and this apparent distance is indicated by $\mathrm{D}^{\prime}$ in Figure 1. The rod extending between the observer and the

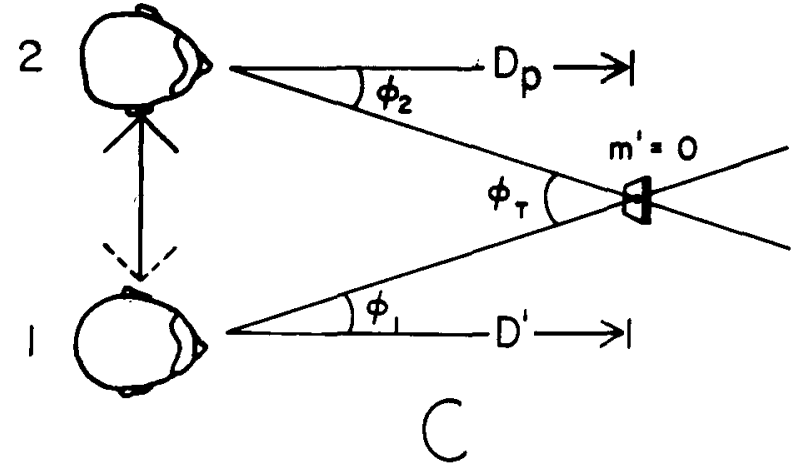

rectangle pivots around a point at a physical distance, $D_{p}$, from the observer as the head is moved between Positions 1 and 2. In Figure $1 A, D_{p}<D^{\prime}$, and, as the head moves from side to side between Positions 1 and 2, the rectangle appears to move laterally in a direction opposite to that of the head motion. In Figure 1B, $D_{p}>D^{\prime}$, and, as the head is moved between Positions 1 and 2, the rectangle appears to move laterally in a direction the same as that of the head motion. Only in the case of Figure $1 C$, in which $D_{p}=D^{\prime}$, will the rectangle appear to be stationary as the head is moved laterally. Thus the pivot distance, $D_{p}$, at which the rectangle appears stationary despite head motion (the null perception) is a measure, albeit an indirect measure, of the perceived distance of the rectangle. More generally, the apparent motion $\left(\mathrm{m}^{\prime}\right)$ of $\cdot$ the object can be expressed as

$$
\mathrm{m}^{\prime}=\mathbf{A}^{\prime}-\phi^{\prime} \mathrm{T}^{\prime},
$$


where $A^{\prime}$ is the sensed motion of the head, $\phi^{\prime} T$ is the sensed turning of the eyes (expressed in radians) in order to maintain fixation on the object as the head is moved, and $D^{\prime}$ is the perceived distance of the object from the observer (Gogel \& Tietz, 1973, 1974). If it can be assumed that $A^{\prime}=A$ and that $\phi^{\prime} \mathrm{T}=\phi_{\mathrm{T}}$, it follows that whenever $\mathrm{m}^{\prime}=0$ (the null perception), $D_{p}$ will equal $D^{\prime}$.

To avoid the mechanical problems involved in the length of rod required to produce large values of $D_{p}$, the actual apparatus used a system of two levers, as shown in Figure 2 and as described more fully elsewhere (Gogel \& Newton, 1976). Figure 2 shows the physical position and physical motion of a rectangle $(E)$ as the head is moved between Positions 1 and 2 . Instead of a long rod pivoted at $D_{p}$, a shorter rigid bar extending from the position of the observer's head to a distance only slightly beyond the physical position, $E$, of the stimulus was used. The motion of the rod and stimulus around $D_{p}$ was accomplished by the two levers labeled $L_{a}$ and $L_{b}$ in Figure 2. Two positions of the levers $L_{a}$ and $L_{b}$ for two head positions and a particular value of $D_{p}$ are shown in the figure. Lever $L_{a}$ pivots around $R_{a}$ as

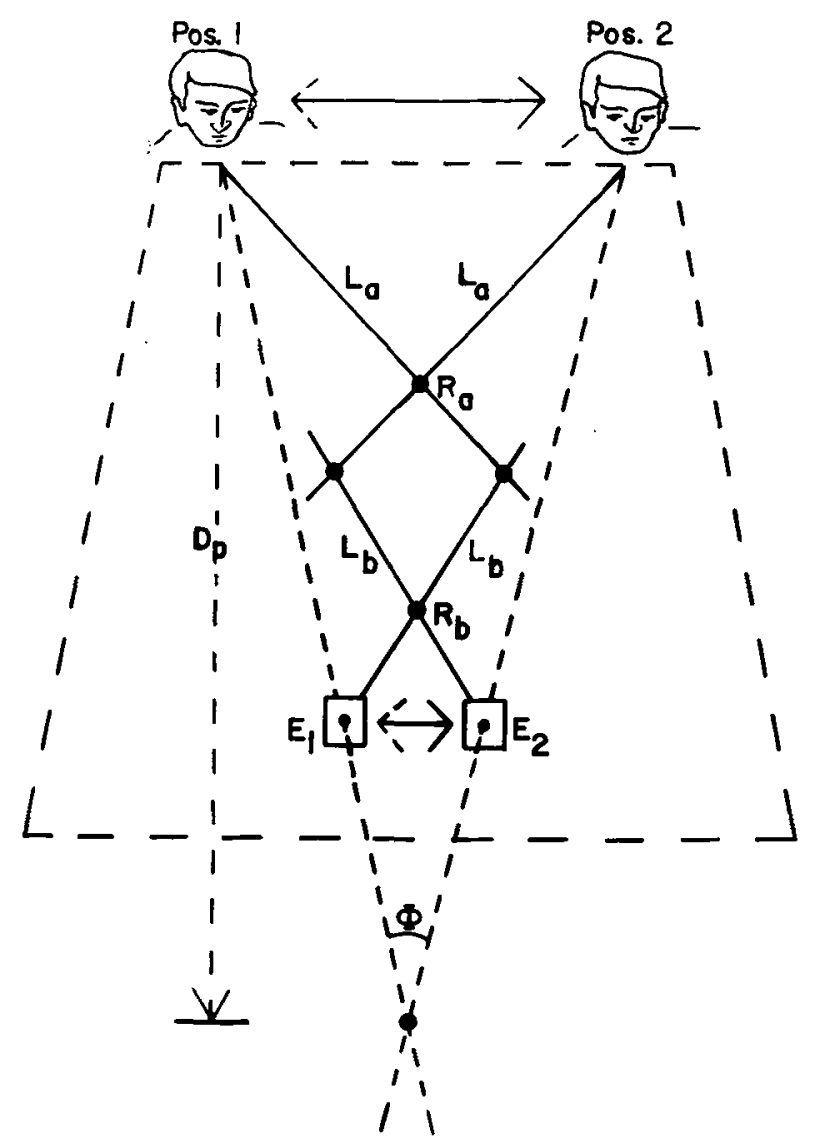

Figure 2. A schematic representation of an apparatus for producing a continuously adjustable distance $\left(D_{p}\right)$ around which the line of sight to Object $E$ will pivot. the head is moved. The rotation of $\mathrm{L}_{\mathrm{a}}$, in turn, causes lever $L_{b}$ to rotate around $R_{b}$, with the result that the rectangle moves physically between $E_{1}$ and $E_{2}$ as the head is moved between Positions 1 and 2. Pivot $R_{a}$ can be moved toward or away from the observer so as to change quantitatively the amount and direction (either with or against the head) of the physical motion of the rectangle between $E_{1}$ and $E_{2}$ as the head is moved between Positions 1 and 2 . With Pivot $R_{\mathbf{a}}$ at a distance closer to the observer than the laterally movable attachment between $L_{a}$ and $L_{b}, E$ will physically move in the same direction as the motion of the head. This is the situation illustrated in Figure 2. With Pivot $R_{a}$ at the distance of the sliding pivot attaching $L_{a}$ to $L_{b}, E$ will remain stationary as the head is moved, and with $R_{a}$ beyond this laterally movable attachment, $E$ will physically move in a direction opposite to the direction of motion of the head. Thus, moving $R_{a}$ increasingly away from or toward the position of the observer will continuously decrease or increase, respectively, the magnitude of $D_{p}$. Regardless of the physical motion of $E$, the adjusted value of $D_{p}$ at which $\mathrm{E}$ no longer appears to move (the null position) is a measure of the perceived egocentric distance of $\mathrm{E}$. This measure of perceived distance will be labeled $D_{m}^{\prime}$. The apparatus permitted $D_{p}$ to be changed continuously from .8 to $35.5 \mathrm{~m}$ by throwing a switch which activated a motor and adjusted the physical position of $R_{a}$. The physical position of $R_{a}$ was measured by a reading on an electronic voltmeter which, after calibration, was converted to values of $\mathrm{D}_{\mathrm{p}}$. Thus, the perceived distances of the object could be determined from the voltmeter reading resulting from the adjustment of Pivot $R_{a}$ at which the object appeared to the observer to be stationary as the head moved back and forth laterally. Since the observer was not aware of the relation between $\mathrm{m}^{\prime}$ and $\mathrm{D}^{\prime}$ expressed by Equation 6, the results obtained from the head motion procedure are assumed to be a pure measure of perceived distance $\left(D^{\prime}\right)$, i.e., a measure of $D^{\prime}$ unmodified by cognitive distance $\left(D_{c}\right)$. Verbal reports of the apparent distances of the stimuli also were obtained in the present study. A comparison between the distance judgments obtained from the head-motion procedure and those obtained by verbal reports of distance permits the evaluation of the contribution of cognitive processes to the verbal reports of the distances of familiar objects.

\section{EXPERIMENT 1}

\section{Apparatus}

\section{Method}

The room in which the experiment was conducted was divided into two visual alleys, each with its own observation position consisting of a head- and chinrest and a viewing aperture and shutter. The observation positions were in a lightproof booth 
with the illumination of each alley independently controlled. A speaker and microphone allowed the experimenter and observer to communicate at each observation position. One of the visual alleys, to be called the experimental alley, contained the headmovement apparatus schematically diagramed in Figure 2 . The experimental alley was lined with black velveteen and was totally dark except for the luminous stimuli to be described. The other visual alley, called the calibration alley, was illuminated throughout its length by overhead incandescent lights. The floor of the calibration alley was formed by a table top, $800 \mathrm{~cm}$ long and $95 \mathrm{~cm}$ wide, which was covered with tan cloth and was located $32 \mathrm{~cm}$ below the level of the observer's eyes. Six white cards (10 cm on a side) stood on the floor of the calibration alley at different distances from the observer, with the nearest card at $40 \mathrm{~cm}$ and the farthest card at $650 \mathrm{~cm}$. A number from 2 to 7 (randomly selected) was painted on each card so that the experimenter could identify each card to the observer during the calibration procedure. The purpose of the calibration alley was to obtain an equation that would correct for observer idiosyncracies in applying the memory of a foot ruler to the verbal estimation of distances (Gogel et al., 1957). This was accomplished by assuming that errors in verbally estimating the distances of the cards on the alley floor could be attributed entirely to errors in the memory or application of a foot ruler to perceived distance. In other words it is assumed, for the purpose of calibration, that no systematic errors occurred in the perception of distance in the calibration alley.'

Five stimulus objects were used with the head-movement apparatus in the experimental alley. One of these, a point of light, was used only to provide practice with the head-movement apparatus prior to presenting the experimental stimuli. One of the experimental stimuli was a luminous rectangle, $10.45 \mathrm{~cm}$ wide $\times 5.22 \mathrm{~cm}$ high, formed by a rectangular opening mounted in front of a luminous surface. The other three experimental stimuli were positive transparencies of familiar objects (a key, a pair of sunglasses, and a guitar). The largest extent (the long axis) of the image on the transparency was $11.35 \mathrm{~cm}$ for the key, $9.60 \mathrm{~cm}$ for the sunglasses, and $10.40 \mathrm{~cm}$ for the guitar. All of the experimental stimuli were mounted with the long axis horizontal in front of the same luminous source. The luminances of the familiar objects were matched to the relative luminances of the objects actually photographed. The luminances of the brightest portions of the transparencies were $.10, .16, .024$, and $.12 \mathrm{fL}$ for the key, sunglasses, guitar, and rectangle, respectively. The stimuli were presented one at a time in an otherwise totally dark environment at a constant distance of $133 \mathrm{~cm}$ from the observer. The stimuli in the experimental alley were always vieweci monocularly with the left eye of the observer occluded. The physical distances at which the actual familiar object would need to be placed to subtend the same size on the eye as the transparency is called the simulated distance of the familiar object. The simulated distance was $63 \mathrm{~cm}$ for the key, $185 \mathrm{~cm}$ for the sunglasses, and $1,236 \mathrm{~cm}$ for the guitar.

The head- and chinrest for the experimental alley could be moved left and right through a distance of $10.5 \mathrm{~cm}$. One end of the rigid bar on which the stimulus was mounted was attached to the head-and chinrest. A spherical lens of +0.75 diopters was also mounted on this bar directly in front of the observer's eyes. This lens placed all of the stimuli accommodatively at optical infinity in order to minimize the effect of oculomotor cues of distance. Two handles attached to the head- and chinrest were grasped by the observer with his left and right hand to move the head- and chinrest assembly laterally while keeping his head in the head- and chinrest. This lateral motion of the head caused the rigid bar extending from the head position to the stimulus to pivot around the distance $D_{p}$, with this distance modifiable by changing the position of $R_{a}$ as shown in Figure 2. Since both the stimulus and the lens were mounted rigidly on the bar, they remained in alignment with the observer's right eye as the observer fixated the stimulus for all lateral positions of the head. The rate at which the observer moved his head back and forth was controlled by the sound of a metronome. The metronome produced a click every $1.86 \mathrm{sec}$, and the observer was instructed to move his head to the extreme right or left in synchrony with the clicks. When making the head motions, the back of the observer's hand contacted a vertical metal plate placed at each end of the path of travel in order to specify the extreme right and left location of the movable head- and chinrest. A white noise from the speaker in the observation booth masked the sound of the apparatus as the head was moved or as the experimenter modified the position of the adjustable pivot.

\section{Observers}

The observers were 48 undergraduates ( 38 women and 10 men) who partially satisfied a course requirement by participating in the experiment. All had a vernier acuity of $20 / 20$ near and far in both eyes, and all were naive regarding the purpose of the experiment.

\section{Procedure}

Experimental situations. General instructions were given to the observer with a model used to illustrate the several tasks, including the task of reporting the direction of the apparent motion of the object relative to the direction of motion of the head. The observers were then taken into the observation booth and, with the lights on and the viewing occluder closed, given practice in moving their heads left and right in the head- and chinrest in time with the clicks of the metronome. Following this, the booth lights were turned off and the shutter covering the viewing aperture was raised, revealing the practice object (the small point of light) in the otherwise totally dark experimental alley. For all observers, the point of light was the first stimulus presented. The instructions were to move the head in the head- and chinrest to the extreme right limit of travel, and upon hearing a click of the metronome to move the head repetitively left and right in time with the metronome clicks for a total of four head movements (two complete left-right movements). The observer was also instructed to fixate the center of the stimulus during the head motion and, if the object appeared to move, to note whether the motion was in the same or in the opposite direction as the motion of the head. Following the four head motions, the shutter was closed and the observer verbally communicated his response of no motion, or of motion with or against the head. The method of limits was used to determine the values of $D_{p}$ that produced no apparent motion in the stimulus (the null position) as the head was moved. Starting with $D_{p}$ very large or very small, the experimenter threw a switch to adjust the $D_{p}$ to predetermined descending or ascending values, with these values indicated to the experimenter by readings on the electronic voltmeter. These adjustments were made with the viewing shutter closed and were completed by the experimenter from a position outside the observation booth. The changes in $D_{p}$ used with this method of limits provided equal changes in the amount of physical motion of the stimulus for a constant motion of the head. The null position was the value of $D_{p}$ (determined from the voltmeter reading) at which the observer's report of stimulus motion changed from motion in one direction to motion in the other direction relative to the head motion or at which the observer reported that no motion was present with a motion in opposite directions reported on each side of this value.

After finding the null position for an ascending series, the null position for a descending series of adjustments was obtained. The average of these adjustments, expressed as $D_{p}$, provided the measure of the perceived distance of the point of light from the observer as determined by the head-movement technique. Since the point of light was a practice stimulus, the results from the presentation of the point were not used. Upon being presented with each of the remaining stimuli (presented successively one at a time), the movable head- and chinrest was immobilized by the experimenter at the center of its travel. This was accomplished from a position outside the booth. With the head- and chinrest assembly fixed in this position, the observer identified the object 
verbally and gave verbal estimates (in feet or inches or in some combination of both) of the perceived distance of the object from himself and of its perceived width. For the width judgments, the observer was instructed to imagine a short vertical line at each side of the widest extent of the object and to indicate the apparent distance between these lines. For half of the observers, the reports of size preceded the reports of distance, and for the remaining observers, the order was reversed. Following the verbal report of the size of the object, the observer was asked whether the object appeared to be normal in size. If the object was reported to be off-sized in appearance, the observer was asked to indicate the size of a normal object of that kind. After completing the verbal reports of size and distance, the head- and chinrest was made mobile and the procedure used in determining the null position of $D_{p}$ for the point of light was repeated with each of the remaining stimuli. The order of the ascending and descending series and the order in which the key, sunglasses, guitar, and rectangle was presented was varied systematically between observers, with each series and each stimulus presented first an equal number of times. Occasionally an observer did not report transition from no motion or motion against the head to motion with the head. In this case, a different observer was used in his place. This occurred with respect to $5,4,15,1$, and 5 observers in Experiments 1, 2, 3, 4, and 5, respectively. It is not clear why the large number of replacements were required in Experiment 3.

Calibration situation. The experimental conditions were always completed before presentation of the calibration situation. The observation during the calibration situation was always binocular. In the calibration situation, the observer was asked to give verbal reports of perceived distance (expressed in feet or inches or in some combination of both) for the six cards, with the experimenter indicating the card by number. The order in which the distance was reported for the six cards followed a repeated Latin-square design.

\section{Results}

The distance results obtained in Experiment 1 by the head-movement procedure and by the method of verbal reports are shown in Table 1 . In the case of the measurements using the head-movement procedure, the distributions of scores $\left(D^{\prime} m\right)$ upon which the results in Table 1 are based were obtained by averaging two null adjustments from each observer, one from the ascending series and the other from the descending series used with the method of limits. The uncalibrated verbal reports $\left(D_{v}\right)$ are the verbal reports as communicated by the observer converted to centimeters. The calibrated verbal reports $\left(D^{\prime} v\right)$ are the result of transforming the uncalibrated verbal reports by the data obtained in the calibration alley. For this purpose, a power function was fitted to the calibration data from each observer, and this calibration equation was used to convert each verbal report obtained in the experimental situations from that observer to a $D^{\prime}{ }_{v}$. The calibration procedure, although reducing the differences among observers occurring as a result of idiosyncracies in mentally applying a foot ruler to a perceived distance in the experimental situations, cannot reduce the cognitive contributions to the verbal reports possibly introduced by the off-sized perceptions in the experimental situations. The power function relating the verbal reports $\left(D_{v}\right)$ to the perceived distance $\left(D^{\prime} v\right)$ obtained by averaging the coefficients and exponents obtained from the individual calibration data is $D_{v}=.654\left(D^{\prime}\right)^{1.04}$. This function is very similar to that obtained using a calibration alley in other studies (Gogel \& Tietz, 1973, 1974). The simulated distances of the familiar objects are shown in the last row of Table 1. These are the values that would be expected if familiar size were a completely accurate determiner

Table 1

Distance Responses Obtained in Experiment 1 from the Head-Movement Procedure (D' $m$ ) and from the Method of Verbal Report ( ${ }^{\prime}{ }_{v}$ )

\begin{tabular}{|c|c|c|c|c|c|c|c|c|}
\hline & \multicolumn{4}{|c|}{ First Trials $(\mathrm{N}=12)$} & \multicolumn{4}{|c|}{ Later Trials $(N=36)$} \\
\hline & Key & Sunglasses & Guitar & Rectangle & Key & Sunglasses & Guitar & Rectangle \\
\hline & \multicolumn{8}{|c|}{ Perceived Distance from Head Motion $\left(D_{m}^{\prime}\right)$} \\
\hline Mean & 206 & 260 & 296 & 281 & 275 & 276 & 324 & 316 \\
\hline Geometric Mean & 201 & 238 & 276 & 263 & 252 & 257 & 299 & 287 \\
\hline Median & 213 & 230 & 273 & 260 & 250 & 239 & 317 & 273 \\
\hline \multirow[t]{2}{*}{ SD } & 46 & 114 & 107 & 108 & 111 & 110 & 127 & 141 \\
\hline & \multicolumn{8}{|c|}{ Verbal Reports of Perceived Distance $\left(D_{v}\right)$} \\
\hline Mean & 38 & 158 & 376 & 397 & 60 & 163 & 423 & 205 \\
\hline Geometric Mean & 35 & 121 & 280 & 222 & 39 & 101 & 220 & 121 \\
\hline Median & 32 & 152 & 305 & 290 & 30 & 99 & 274 & 91 \\
\hline \multirow[t]{2}{*}{$\mathrm{SD}$} & 14 & 115 & 240 & 330 & 59 & 290 & 542 & 214 \\
\hline & \multicolumn{8}{|c|}{ Calibrated Verbal Reports of Perceived Distance $\left(D_{v}^{\prime}\right)$} \\
\hline Mean & 52 & 217 & 470 & 536 & 90 & 211 & 500 & 265 \\
\hline Geometric Mean & 49 & 168 & 370 & 314 & 59 & 148 & 303 & 166 \\
\hline Median & 42 & 187 & 412 & 387 & 56 & 151 & 447 & 172 \\
\hline SD & 21 & 156 & 311 & 469 & 85 & 310 & 447 & 258 \\
\hline Simulated Distance & 63 & 185 & 1236 & & 63 & 185 & 1236 & \\
\hline
\end{tabular}


Table 2

Verbal Reports of Width in Centimeters Obtained in Experiment 1 , with $S_{v}^{\prime}$ the Report of Perceived Width and $S_{c}$ the Report of Remembered Familiar Width $(\mathbf{N}=48)$

\begin{tabular}{|c|c|c|c|c|c|c|c|c|c|c|}
\hline & \multicolumn{3}{|c|}{ Key } & \multicolumn{3}{|c|}{ Sunglasses } & \multicolumn{3}{|c|}{ Guitar } & \multirow{2}{*}{$\frac{\text { Rectangle }}{\mathrm{S}_{\mathrm{v}}}$} \\
\hline & $S_{v}^{\prime}$ & $s_{c}$ & $\mathrm{~S}_{\mathrm{v}}^{\prime} / \mathrm{S}_{\mathrm{c}}$ & $S_{v}^{\prime}$ & $S_{c}$ & $\mathrm{~S}_{\mathrm{v}}{ }_{\mathrm{v}} / \mathrm{S}_{\mathrm{c}}$ & $s_{v}^{\prime}$ & $\mathrm{S}_{\mathrm{c}}$ & $S_{v}^{\prime} / S_{c}$ & \\
\hline Mean & 13.3 & 4.8 & 3.0 & 30.2 & 13.9 & 2.4 & 32.9 & 100.8 & 0.3 & 54.8 \\
\hline Geometric Mean & 6.5 & 4.6 & 1.4 & 12.3 & 13.7 & 0.9 & 15.3 & 98.7 & 0.2 & 17.2 \\
\hline Median & 5.2 & 5.2 & 1.0 & 12.8 & 15.2 & 1.0 & 12.8 & 91.4 & 0.1 & 11.4 \\
\hline SD & 35.1 & 1.6 & 7.0 & 88.8 & 2.7 & 8.6 & 40.0 & 21.7 & 0.4 & 92.8 \\
\hline Physical & 11.4 & 5.4 & 2.1 & 9.6 & 13.4 & 0.7 & 10.4 & 96.5 & 0.1 & 10.4 \\
\hline
\end{tabular}

(either perceptual or cognitive) of the perceived distance of a normal-sized object of that particular kind from the observer. It will be assumed that the geometric means best represent the data and, since the distributions of distance responses are sometimes skewed, the data were converted to logarithms before being analyzed.

The verbal reports $\left(\mathbf{S}^{\prime}{ }^{\prime}\right)$ of apparent width, the reported memory of familiar size $\left(S_{c}\right)$, and the ratio of, the averages of these is given in Table 2 for the familiar objects. It seems from these results that, if the reported size of the stimulus was different from its normal size, it was reported more often to be larger rather than smaller for the key and more often to be smaller rather than larger for the guitar. The results expected in Table 2 if $S^{\prime}{ }_{v}$ were equal to the physical width of the image on the transparency and $\mathrm{S}_{\mathrm{c}}$ were equal to the physical width of a normal-sized familiar object of that type are shown in the last row of Table 2.

A simple analysis of variance of the differences between the results from the different familiar objects regardless of whether the response to the particular stimulus was obtained on the first, second, third, or fourth trial was significant for both $D^{\prime}{ }_{m}$ and $D^{\prime}{ }_{v}$, $F(2,94)=13.59$ and $74.55, p<.001$. Clea:ly, the simulated distance of the familiar object had an effect upon its perceived distance as measured by either the head-movement or verbal report method. In Table 1, the overall results are separated depending upon whether the perceived distance measure on that particular object was obtained on the first trial on which any of the experimental stimuli were presented to that observer or on the remaining (second, third, or fourth) trials. The purpose of this is to distinguish between familiar size as a cue to the distance of the object from the observer (an egocentric distance) and familiar size as a cue to the distance between successive presentations of the different familiar objects independently of the position of the observer (exocentric distance). Familiar size is a cue to egocentric distance if the differences between the first trials (as a function of the familiar size) are significant (Gogel, 1969; Gogel et al., 1957). Familiar size is a cue to exocentric distance, but not to ego- centric distance if the differences in the distance responses occur only on the remaining trials (not on the first trials) or only between successive trials. The similarity of the results obtained from the first and the remaining trials in Table 1 suggests that the distance results obtained throughout the experiment apply to familiar size as a cue of egocentric distance. This conclusion must be tentative, however, since the number of observations involved in the first trials is small $(\mathrm{N}=12)$.

Since off-sized perceptions often occurred in Experiment 1, it is expected, consistent with Equation 2, that $D_{c} \neq D^{\prime}$. Thus, to the extent that $D_{c}$ contributed to the verbal report (despite the instructions to report only the apparent distance of the objects), $D^{\prime}{ }_{v}$ would be modified in the direction of the simulated distance from familiar size. On the other hand, $\mathrm{D}^{\prime} \mathrm{m}$, which is unaffected by $\mathrm{D}_{\mathrm{c}}$, provides a pure measure of perceived distance. ${ }^{2}$ If $D^{\prime} m$ and $D^{\prime}{ }_{v}$ differ, it follows that this difference can be considered to represent the effect of $D_{c}$ upon the verbal report of apparent distance. It is clear from the $D_{m}^{\prime}$ data of Table 1 that, although the simulated distance of the familiar object modified the perceived distance, this effect was small. The much larger effect of simulated distance on the $\mathrm{D}^{\prime}{ }_{\mathrm{v}}$ data indicates that most (but not all) of the effect of familiar size upon the verbal reports of distance is cognitive, not perceptual, in origin. Also, it will be noted that the greatest difference between the head-movement and verbal report methods of measuring apparent distance with the familiar objects occurred with the smallest simulated distance (produced by the key). Perhaps cognitive factors of correction apply most readily to near distances. Or perhaps, in the case of the guitar, the off-sized perceptions are too extreme (see Table 2) to be used by the observer in the modification of the verbal report by $D_{c}$. An analysis of variance using the logarithmic transformation of the $D^{\prime}{ }_{v}$ and $D_{m}^{\prime}$ data independent of the order of presentation provides clear evidence that the change in perceived distance was greater for $D^{\prime}{ }_{v}$ than for $D^{\prime}$ m with $F(2,94)=$ $51.6, \mathrm{p}<.001$.

Since the rectangle had no specific familiar size, the effect of cognitive factors on the verbal reports 
of the distance of the rectangle should have been minor. In other words, the distance responses to the rectangle from the head-movement technique and the calibrated verbal reports would be expected to be similar. This expectation is not supported by the geometric means of Table 1 if the results of the second through fourth trials (the later trials) rather than first trials are considered. The difference between the response to the rectangle when presented on the first and on later trials may indicate that the prior presentations of a familiar object has a cognitive effect on the verbal report of the distance of the rectangle presented later.

\section{ADDITIONAL EXPERIMENTS (2-4) USING THE KEY AND GUITAR}

The small number of observations obtained from the trials in which a particular familiar object was the first familiar object presented permitted no firm conclusion to be reached in Experiment 1 as to whether the changes in $D^{\prime} m$ as a function of simulated distance indicated that familiar size was a perceptual cue to egocentric, as distinct from exocentric, distance. Experiment 2 added to the data that could be applied to this problem.

Since the transparencies approximated the relative luminance of the actual objects, the average luminances of the different transparencies were different. If the luminance of an object is a cue to perceived distance, these luminance differences between transparencies could have contributed to the change in $D^{\prime}{ }_{m}$ between the familiar objects. This possible factor was examined in Experiment 3.

In Experiment 1, the verbal report of the apparent size and distance of a stimulus always was obtained before using the head-movement procedure. It seemed unlikely that the verbal reports would influence the results obtained with the head-movement procedure, whereas an effect of the reverse order might have been found. In Experiment 4, however, the head- motion procedure always was used prior to obtaining the verbal reports of distance.

\section{Experiment 2 \\ Procedure}

This experiment was identical to Experiment 1, with two exceptions. (1) There were 24 observers, 19 women and 5 men, none of whom had been in Experiment 1. (2) Only the key and guitar transparencies of Experiment 1 were used in Experiment 2.

\section{Experiment 3}

This experiment was identical to Experiment 2, with two exceptions. (1) There were 48 observers, 32 women and 16 men, none of whom had been used in the two previous experiments. (2) The brightest extended portions of the key and guitar stimuli were matched in luminance at .054 $\mathrm{fL}$.

\section{Experiment 4}

This experiment was identical to Experiment 3, with two exceptions. (1) There were 24 observers, 16 women and 8 men, none of whom had been used in any of the previous experiments. (2) The observers used the head-movement procedure with each stimulus presentation before giving the verbal reports.

\section{Results and Discussion}

The geometric means of the distance and size responses from Experiments 2, 3, and 4 are shown in Tables 3 and 4, respectively, with the geometric means from Experiment 1 included for comparison. The number of observers involved in the geometric means of the "first" and "later" trials was 12 and 36 for Experiment 1, 24 and 24 for Experiment 3, and 12 and 12 for Experiments 2 and 4 . Table 3 in general supports the conclusion that, for both the headmovement procedure and the method of verbal report, the distance response for the guitar was greater than that for the key for both first and successive presentations. The significance of the guitar-key differences was evaluated by a simple analysis of variance, with the first and all trials analyzed separately. Consider the $D^{\prime} m$ and $D^{\prime}{ }_{v}$ results from the first presentations for the head-movement procedure. Consistent with the purpose of Experiment 2, the $D^{\prime}{ }_{m}$ data from the trials in which the key or the guitar were presented first in Experiments 1 and 2

Table $\mathbf{3}$

Geometric Means of Distance Responses in Centimeters Obtained in Experiments 1-4 Using the Head-Movement Procedure and the Method of Verbal Report for the Key and Guitar

\begin{tabular}{|c|c|c|c|c|c|c|c|c|}
\hline & \multicolumn{4}{|c|}{ Head-Movement Procedure $\left(D_{m}^{\prime}\right)$} & \multicolumn{4}{|c|}{ Calibrated Verbal Report $\left(D^{\prime} v\right.$ ) } \\
\hline & \multicolumn{2}{|c|}{ First Trials } & \multicolumn{2}{|c|}{ Later Trials } & \multicolumn{2}{|c|}{ First Trials } & \multicolumn{2}{|c|}{ Later Trials } \\
\hline & Key & Guitar & Key & Guitar & Key & Guitar & Key & Guitar \\
\hline Experiment 1 & 201 & 276 & 252 & 299 & 49 & 370 & 59 & 303 \\
\hline Experiment 2 & 261 & 372 & 327 & 482 & 61 & 378 & 65 & 387 \\
\hline Experiment 3 & 264 & 368 & 284 & 372 & 80 & 336 & 55 & 356 \\
\hline Experiment 4 & 317 & 318 & 276 & 458 & 84 & 410 & 50 & 351 \\
\hline Average & 261 & 334 & 285 & 403 & 69 & 374 & 57 & 349 \\
\hline Obtained Ratio & \multicolumn{2}{|c|}{1.3} & \multicolumn{2}{|c|}{1.4} & \multicolumn{2}{|c|}{5.4} & \multicolumn{2}{|c|}{6.1} \\
\hline Simulated Ratio & \multicolumn{2}{|c|}{19.6} & \multicolumn{2}{|c|}{19.6} & \multicolumn{2}{|c|}{19.6} & \multicolumn{2}{|c|}{19.6} \\
\hline
\end{tabular}


Table 4

Geometric Means of Verbal Reports of Width in Centimeters Obtained in Experiments 1-4, with $S^{\prime}$ the Report of Perceived Width of the Stimulus and $S_{c}$ the Report of the Remembered Familiar Width

\begin{tabular}{|c|c|c|c|c|c|c|}
\hline & \multicolumn{3}{|c|}{ Key } & \multicolumn{3}{|c|}{ Guitar } \\
\hline & $\mathbf{S}^{\prime}{ }_{v}$ & $S_{c}$ & $\mathrm{~S}_{\mathbf{v}}^{\prime} / \mathrm{S}_{\mathrm{c}}$ & $\mathbf{S}_{\mathbf{v}}^{\prime}$ & $\mathrm{S}_{\mathbf{c}}$ & $\mathrm{S}_{\mathrm{v}}^{\prime} / \mathrm{S}_{\mathrm{c}}$ \\
\hline Experiment 1 & 6.5 & 4.6 & 1.4 & 15.3 & 98.7 & 0.2 \\
\hline Experiment 2 & 7.2 & 4.5 & 1.6 & 19.3 & 95.0 & 0.2 \\
\hline Experiment 3 & 7.8 & 5.3 & 1.5 & 13.4 & 92.4 & 0.1 \\
\hline Experiment 4 & 5.0 & 4.4 & 1.1 & 17.0 & 87.4 & 0.2 \\
\hline
\end{tabular}

were used together. These combined data, $F(1,46)=$ $5.68, p<.025$, and the first-presentation, head-motion data of Experiment 3, $F(1,46)=7.07, p<.025$, but not of Experiment 4, $F(1,22)<1.0$, were statistically significant. Consider the distance results from the first presentation for the calibrated verbal reports. These were significantly different for the guitar and key, $F(1,46)=78.22, p<.001$, for the combined results from Experiments 1 and 2 and for the results from Experiment 3, $F(1,46)=15.54$, $\mathrm{p}<.001$, and Experiment $4, \mathrm{~F}(1,22)=15.78$, $\mathrm{p}<.001$. Clearly, both the head-movement procedure and the method of verbal report indicate that, on first presentations, familiar size is a cue to egocentric distance. Also, the general similarity of the $D^{\prime}{ }_{m}$ results obtained when the familiar object was presented on first or subsequent trials (second trials in Experiments 2, 3, and 4) indicates that the difference between the geometric means from the key and guitar throughout this study can be attributed to familiar size as a cue to egocentric distance. In addition, the general similarity of the results from the different experiments indicates that the perceived distance effects attributed to familiar size were not due to luminance differences between the familiar objects or to the order in which the head-movement and verbal measures were obtained.

The results from Experiments 2, 3, and 4 support the conclusions from Experiment 1 . As shown in Table 3, the average geometric mean of $D_{m}^{\prime}$ (with equal weight given to each experiment) obtained from the guitar is about 1.3 times as large as that obtained from the key. It can be concluded that the familiar size cue to distance modified somewhat the perceived distance of the object. But the much larger average ratio of the geometric means of $D^{\prime}{ }_{v}$ (about 6 to 1) obtained from these objects indicates that the verbal reports of distance were strongly influenced by a cognitive factor. This cognitive factor seemed to modify the verbal report of the distance of the key more than of the guitar. As shown in Table 4, the key was sometimes perceived as a large off-sized object and the guitar was often perceived as a very small off-sized object. Considering all the 144 observers in the four experiments, 50 reported the key as larger than normal size, 85 as normal size, and 9 as smaller than normal. The corresponding frequencies for the guitar were 16, 20, and 108. As in Experiment 1, an analysis of variance using the logarithmic transformation of the $D^{\prime}{ }_{v}$ and $D^{\prime}{ }_{m}$ data independent of the order of presentation provides clear evidence $(p<.001)$ that the change in perceived distance was greater for $D^{\prime}{ }_{v}$ than for $D^{\prime}{ }_{m}$, with $F(1,23)=23.6$, $F(1,47)=39.4$, and $F(1,23)=26.7$ from Experiments 2, 3, and 4, respectively. Also, considering all of the experimental results together, the ratio of the perceived distance of the key and guitar was greater for $D^{\prime}{ }_{v}$ than for $D^{\prime} m$ for 127 out of the 144 observers, with the reverse occurring for 16 observers and with no difference occurring for one observer.

\section{EXPERIMENT 5}

The greater differences between the key and guitar obtained from the verbal reports as compared with the head-movement technique is attributed to the modification of the verbal reports by the cognitive factor resulting from the off-sized perceptions. The purpose of Experiment 5 was to examine the sensitivity of the head-movement technique by applying it to a situation in which clear differences in perceived distance would be expected to occur. In Experiments 1 through 4 , the objects presented successively were different familiar objects, e.g., a key and guitar. If two retinal sizes of the same familiar object are presented successively, a relative size cue is available between the successive presentations, with the result that the perceived depth between the second presentations will be greater than the perceived depth between the first presentations. As previous research has demonstrated, this relative size cue is a perceptual (not a cognitive) cue of exocentric distance (Gogel \& Tietz, 1974) and its effect should be reflected in the $D^{\prime}{ }_{m}$ as well as in the $D^{\prime}{ }_{v}$ measures. To illustrate the effect of the relative size cue occurring between successive presentations, consider the case in which a normal- and double-sized playing card are successively presented at a constant physical distance using two independent groups of observers. One group first views the normal-sized and then the double-sized card. With the other group, the double-sized card is presented first, followed by the normal-sized card. Suppose also that the familiar size cue to perceived egocentric distance is not very effective, so that both groups perceive the initial presentation at about the same distance, e.g., at about $10 \mathrm{ft}$. According to the relative size cue, the group with the normal-sized card presented first should perceive the double-sized card presented second at a nearer distance than $10 \mathrm{ft}$, e.g., at $5 \mathrm{ft}$. The group viewing the double-sized card first should perceive the normal-sized card presented second at a farther distance than $10 \mathrm{ft}$, e.g., at $20 \mathrm{ft}$. Thus to the extent that familiar size is 
Table 5

Distance Responses in Centimeters Obtained in Experiment 5 from the Head-Movement Procedure $\left(D^{\prime}{ }_{m}\right.$ ) and from the Calibrated Verbal Reports ( $D^{\prime}$ )

\begin{tabular}{|c|c|c|c|c|c|c|c|c|}
\hline & \multicolumn{4}{|c|}{ Head Movement Data $\left(D_{m}^{\prime}\right)$} & \multicolumn{4}{|c|}{ Calibrated Verbal Report $\left(D^{\prime}\right)$} \\
\hline & \multicolumn{2}{|c|}{ First Trial } & \multicolumn{2}{|c|}{ Second Trial } & \multicolumn{2}{|c|}{ First Trial } & \multicolumn{2}{|c|}{ Second Trial } \\
\hline & Large & Small & Large & Small & Large & Small & Large & Small \\
\hline Mean & 277 & 356 & 232 & 401 & 346 & 192 & 77 & 283 \\
\hline Geometric Mean & 252 & 298 & 214 & 356 & 100 & 154 & 60 & 213 \\
\hline Median & 239 & 273 & 186 & 356 & 81 & 194 & 62 & 226 \\
\hline SD & 125 & 276 & 119 & 241 & 1031 & 115 & 68 & 224 \\
\hline Simulated Distance & 89 & 222 & 89 & 222 & 89 & 222 & 89 & 222 \\
\hline
\end{tabular}

not an adequate cue to perceived egocentric distance on the first presentation, the relative size cue between presentations should result in the difference between the perceived distance of the large and small card being greater on the second as compared with the first presentations.

\section{Procedure}

Experiment 5 was identical to Experiment 4 with three exceptions. (1) There were 40 observers, 25 women and 15 men, none of whom had been in any of the previous experiments. (2) The experimental stimuli were two sizes of transparencies of a playing card (10 of clubs). The small transparency measured $3.46 \times$ $5.35 \mathrm{~cm}(60 \%$ of normal size $)$ and the large one $8.66 \times$ $13.30 \mathrm{~cm}$ (150\% of normal size). As in the previous experiments, the stimuli were physically mounted on the arm of the headmovement apparatus at a constant distance of $133 \mathrm{~cm}$ and were viewed through the positive lens to place them at a far accommodative distance. The small transparency (card) simulated a normal sized card at $222 \mathrm{~cm}$, and the large transparency simulated a normal sized card at $88.9 \mathrm{~cm}$. The luminances of the white portions of the two stimuli were matched at $.12 \mathrm{fL}$. (3) The observers were instructed to report the apparent height (not the apparent width) of the upright card.

\section{Results and Discussion}

The results from the head-movement procedure $\left(D^{\prime} \mathrm{m}\right)$ and the calibrated verbal reports of distance $\left(D^{\prime}{ }_{v}\right)$ are shown in Table 5, with the size judgments summarized in Table 6 . It seems from Table 6 that at least the small card tended to be seen as a small off-sized object-in this case, as a smaller than normal playing card. The verbal report of the height of the physically large card was greater than, equal to, or less than that of the physically smaller card for 29,6 , and 5 observers, respectively. This is consistent with the effect expected from a tendency to perceive the playing cards as less different in distance than would be expected from the difference in their simulated distances. Consider the $D^{\prime} m$ and $D^{\prime}{ }_{v}$ geometric means of the first presentations of Table 5 . Although the $D^{\prime} m$ and $D^{\prime}{ }_{v}$ of the small card was greater than that of the large card on the first trials, neither of these differences was significant at the .05 level, $F(1,38)=1.11$ and 1.79. It seems that the difference in the simulated distances between the large and small cards was not sufficient to produce a significant $D^{\prime} m$ or $D^{\prime}{ }_{v}$ difference on the first trials.
On the second trials, both the $D^{\prime} m$ and $D^{\prime}{ }_{v}$ were significantly different (beyond the .01 level) between the two card sizes, $F(1,38)=14.79$ and 27.71 . It can be concluded that the head-movement procedure as well as the method of verbal report is sensitive to the changes in perceived distance expected from the relative size cue occurring between successive presentations. This result supports the conclusion that the lesser relation between perceived and simulated distance obtained from the head movement as compared with the verbal reports in the previous experiments is not a result of any insensitivity of the headmovement procedure in measuring perceived distance, but instead indicates that much of the differences obtained with the verbal reports on the first trials can be attributed to the cognitive effect of the off-sized perceptions.

\section{DISCUSSION}

There is no doubt that the measured perceived distance varied as a function of the simulated distance of the familiar object in this study, using either the head-movement or the verbal report procedure. Considering the data from all trials, both the $D^{\prime} m$ and $D^{\prime}{ }_{v}$ results were significantly different for the key and guitar in each of Experiments 1 through 4 at (at least) the .01 level. Considering all four experiments together, the number of observers who indicated that the guitar was more distant, equal in distance, or less distant than the key was 108, 19, and 17 for $D^{\prime} m$ and 129,7 , and 8 for $D^{\prime}{ }_{v}$, respectively.

Table 6

Verbal Reports of Height in Centimeters Obtained In Experiment 5, with $S^{\prime}$ the Report of Perceived Height of the Playing Card and $S_{c}$ the Report of the Remembered Familiar Height

\begin{tabular}{|c|c|c|c|c|c|}
\hline & \multicolumn{2}{|c|}{$\mathbf{S}_{\mathbf{v}}$ of Card } & \multirow{2}{*}{$\begin{array}{c}\text { Assumed } \\
\text { Size } \\
\left(\mathrm{S}_{\mathrm{c}}\right)\end{array}$} & \multicolumn{2}{|c|}{$S_{v}^{\prime} / S_{c}$} \\
\hline & Large & Small & & Large & Small \\
\hline Mean & 19.8 & 9.5 & 9.2 & 2.2 & 1.0 \\
\hline Geometric Mean & 9.4 & 6.0 & 9.0 & 1.0 & 0.7 \\
\hline Median & 7.6 & 5.2 & 10.1 & 0.8 & 0.5 \\
\hline SD & 49.2 & 11.1 & 1.6 & 29.8 & 6.7 \\
\hline
\end{tabular}


According to the $D^{\prime} m$ results, the perceived distance of the familiar objects was displaced from their simulated distances toward the 2-3-m distance of the specific distance tendency. As a consequence of this effect of the specific distance tendency, the familiar objects were often perceived as off-sized, with the key sometimes reported as larger than normal and the guitar usually reported as smaller than normal. These off-sized perceptions in agreement with Equation 2 produced a $D_{c}$ different from $D^{\prime}$, and, as a result, $D^{\prime}{ }_{v}$ showed a larger variation than $D^{\prime} m$ with simulated distance. Since the observer was asked to report apparent distance, the effect of $D_{c}$ upon $D^{\prime} v$ occurred despite the "apparent" instructions. It seems that instructions are only one of the factors determining the demand characteristics of the experiment. It should be noted that the head-movement procedure, by providing an unbiased measure of perceived distance, also provides a criterion against which the effect of instructions can be evaluated.

It can be concluded from this study that, although familiar size is a cue to perceived distance, it is not a very robust cue since it is readily modified by the specific distance tendency. The stronger relation between apparent and simulated distance from familiar objects usually obtained by the method of verbal report is produced by a cognitive source of information resulting from perceptions of the familiar object as larger or smaller than normal.

\section{REFERENCES}

Carlson, V. R. Overestimation in size-constancy judgments. American Journal of Psychology, 1960, 73, 199-213.

CARLSON, V. R. Size-constancy judgments and perceptual compromise. Joumal of Experimental Psychology, 1962, 63, 68-73.

Epstein, W. The known size-apparent distance hypothesis. American Journal of Psychology, 1961, 74, 333-346.

EPSTEIN, W. Attitudes of judgment and the size-distance invariance hypothesis. Journal of Experimental Psychology, 1963, 66, 78-83.

EPSTEIN, W. Varieties of perceptual learning. New York: McGrawHill, 1967.
Foley, J. M., \& Held, R. Visually directed pointing as a function of target distance, direction and available cues. Perception \& Psychophysics, 1972, 11, 423-427.

GoGEL, W. C. The effect of object familiarity on the perception of size and distance. Quarterly Journal of Experimental Psychology, $1969,21,239-247$.

Gogel, W. C. Cognitive factors in spatial responses. Psychologia, 1974, 17, 213-225.

Gogel, W. C., Hartman, B. O., \& Harker, G. S. The retinal size of a familiar object as a determiner of apparent distance. Psychological Monographs, 1957, 71(Whole No. 422), 1-16.

Gogel, W. C., \& Mertens, H. W. Perceived size and distance of familiar objects. Perceptual and Motor Skills, 1967, 25, 213-225.

Gogel, W. C., \& Newron, R. E. Perception of off-sized objects. Perception \& Psychophysics, 1969, 5, 7-9.

Gogel, W. C., \& Newton, R. E. An apparatus for the indirect measurement of perceived distance. Perceptual and Motor Skills. $1976,43,295-302$.

Gogel, W. C., \& Tretz, I. D. Absolute motion parallax and the specific distance tendency. Perception \& Psychophysics, 1973, 13, 284-292.

GoGel, W. C., \& TIETz, J. D. The effect of perceived distance on perceived movement. Perception \& Psychophysics, 1974, 16, 70-78.

\section{NOTES}

1. The application of a head-motion technique to the measurement of possible errors in perceived distance occurring in full-cue conditions of observation will be discussed in a later publication.

2. To say that $D_{m}^{\prime}$ is unaffected by $D_{c}$ is a strong statement. It should be noted, however, that the $D_{c}$ being discussed results from the perception of the familiar objects as off-sized. It is possible that adjusting the pivot distance until the stimulus does not appear to move could be modified to some degree by cognitive effects. These would be cognitive effects associated with maintaining the criterion of no apparent motion; they would not involve cognitive effects from off-sized perceptions, nor, more generally, would they involve cognitive effects associated with responding to a perceived distance. To appreciate this, recall that with the adjustable pivot procedure the observer is not asked anything about perceived distance. Instead, he is asked to adjust a switch until the object does not appear to move as he moves his head. It is difficult to imagine that any inferences that the observer might have about the distance of the object could in any way influence this null adjustment.

(Received for publication May 17, 1976; revision accepted September 16, 1976.) 